\title{
High resolution spectroscopy of the galactic candidate LBV MWC 314*
}

\author{
A.S. Miroshnichenko ${ }^{1,2}$, Y. Frémat ${ }^{3, \star \star}$, L. Houziaux ${ }^{3}$, Y. Andrillat $^{4}$, E.L. Chentsov ${ }^{5}$, and V.G. Klochkova ${ }^{5}$ \\ 1 Dept. of Physics \& Astronomy, Univeristy of Toledo, Toledo, OH 43606-3390, U.S.A. \\ e-mail: anatoly@physics.utoledo.edu \\ 2 Central Astronomical Observatory of the Russian Academy of Sciences at Pulkovo, Saint-Petersburg, 196140, Russia \\ 3 Université de Mons-Hainaut, Groupe d'Astrophysique et de Spectroscopie, 20 place du Parc, B-7000 Mons, Belgium \\ 4 Laboratoire d'Astronomie, Université de Montpellier 2, URA 1280 and 1981, CNRS, Place Eugène Bataillon, 34095 Monpellier \\ Cedex 5, France \\ 5 Special Astrophysical Observatory of the Russian Academy of Sciences, Karachai-Cherkess Republic, Nizhnyi Arkhyz, 357147, \\ Russia
}

Received February 5; accepted March 20, 1998

\begin{abstract}
The results of high-resolution optical spectroscopy obtained for a recently suggested LBV candidate, MWC 314, are presented. Photospheric lines mostly of $\mathrm{N}$ II and S II have been found for the first time with a resolution of $0.4-0.8 \AA$. They imply a N/O overabundance previously reported for AG and HR Car since no O II absorptions were detected. Nearly 400 emission lines have been identified in the spectrum between 4190 and $8864 \AA$ many of which appear double-peaked. No significant $\mathrm{P}$ Cyg-type absorption components have been found for the optically thick Balmer lines. This suggests that the stellar wind of MWC 314 is non-spherical and the circumstellar envelope is viewed not edge-on. The systemic velocity measured using the emission lines of ionized metals turned out to be $+55 \mathrm{~km} \mathrm{~s}^{-1}$, which corresponds to a distance towards the object of $3.0 \pm 0.2 \mathrm{kpc}$, according to the galactic rotation curve. Our analysis of the characteristics of both photospheric and wind lines resulted in an estimate of the object's $T_{\text {eff }}$ of nearly $25000 \mathrm{~K}$, which, however, needs further improvement. The other stellar parameters were found to be as follows: $\log L_{\mathrm{bol}} / L \odot=6.1 \pm 0.3$, $R_{*}=60_{-10}^{+30} R \odot$. Our study confirms that MWC 314 is one of the most luminous stars in the Milky Way.
\end{abstract}

Key words: stars: early-type; supergiants; abundances stars: mass loss — stars: individual: MWC 314

Send offprint requests to: A.S. Miroshnichenko

* Tables 1-4 are available in electronic form at the CDS via anonymous ftp 130.79.128.5

$\star \star$ FRIA fellow.

\section{Introduction}

MWC $314=\mathrm{BD}+14^{\circ} 3887$ was discovered by Merrill (1927), who found hydrogen and Fe II emissions in its spectrum. Swensson (1942) detected the interstellar H and K Ca II lines and $4430 \AA$ band, Balmer emissions from $\mathrm{H} \alpha$ to $\mathrm{H}_{8}$, emissions of $\mathrm{Na}$ I at 5890 and $5896 \AA$, and, finally, numerous emissions of metallic lines including $\mathrm{Fe}$ II, $\mathrm{Cr}$ II, [Fe II], and two lines of [Fe III]. Photospheric lines and spectral features of late-type stars were not observed in these, rather low-resolution, spectra. The first photoelectric $U B V$ photometry obtained by Hiltner (1956) showed that the star was extremely red $\left(B-V \sim 1^{\mathrm{m}} 6\right)$, but with $U-B \sim 0.3$ it is located at the reddening line for earlyB stars. Near-IR photometry obtained by Allen (1973) implies a small excess radiation in this region. Recently Miroshnichenko (1996) reported results of a new photometric and spectroscopic study of MWC 314. It was based on nearly 50 photometric $U B V R I$ and $U B V R I J H K$ observations obtained in 1990-1994, two medium-resolution spectra $\left(4000-7500 \AA\right.$, dispersion $50 \AA \mathrm{mm}^{-1}$, resolution $2 \AA$ ) obtained at the 6 -meter telescope of the Russian Academy of Sciences in 1989 and 1991, the IUE lowresolution spectra obtained in 1984 and 1992, and the IRAS photometry at 12 and $25 \mu \mathrm{m}$. He found that the object is variable in all photometric bands with a mean amplitude of 0 . 3 . Analysing the spectral energy distribution (SED) and Balmer emission line profiles he concluded that MWC 314 is a highly reddened supergiant with $A_{V}=5{ }^{\mathrm{m}} 7$ and the following main parameters: $\log L / L_{\odot}=6.2, T_{\text {eff }}=$ $30000 \mathrm{~K}, R_{*}=50 R_{\odot}$. The strong and rather narrow Balmer lines indicate that the star have a high mass loss rate: $\dot{\mathrm{M}}=310^{-5} M_{\odot} \mathrm{yr}^{-1}$. The wind terminal velocity, $v_{\infty}=500 \mathrm{~km} \mathrm{~s}^{-1}$, is much lower than in normal 
supergiants. All these findings led Miroshnichenko to the suggestion that MWC 314 is a candidate LBV, the second one in the northern hemisphere after $\mathrm{P}$ Cyg.

MWC 314 is not an ordinary supergiant. It displays very strong Fe II emission lines which are also significant features of the spectra of B[e] stars (e.g., Andrillat et al. 1996). However, it has not been previously recognized as a $\mathrm{B}[\mathrm{e}]$ star because very few forbidden lines have been so far identified in its spectrum and furthermore there is no evidence of circumstellar dust as its IR-excess is very small.

Miroshnichenko (1996) noted that some problems remained with this object. In particular, high-resolution spectroscopy was needed to obtain more detailed emission line profiles and to detect eventual photospheric lines. The stellar temperature estimate was based mainly on the SED in the UV region since Balmer line profiles were not very sensitive to the temperature changes. Moreover, He II lines have not been detected in the previous spectra indicating that $T_{\text {eff }}<(26-27) 10^{3} \mathrm{~K}$ (Schmutz et al. 1991).

Recently, a series of papers reporting results of highresolution spectroscopic observations of strong emissionline B[e] stars has been published (Jaschek et al. 1996a,b; Andrillat et al. 1996, 1997). The present paper is aimed to make further progress in the understanding of a similar object. Here we report the results of a new, more highdispersion, spectroscopy of MWC 314 and its analysis.

\section{Observations}

The observations were obtained at the 1.52-meter telescope of the Observatoire de Haute Provence (OHP) on 1996 June 12 and from 1997 July 20 to 1997 July 27 and at the 6-meter telescope of the Special Astrophysical Observatory (SAO) of the Russian Academy of Sciences on 1997 July 23 (JD 2450653.46) and on 1997 November 22 (JD 2450775.16).

The AURELIE spectrograph (Gillet et al. 1994) was mounted at the Coudé focus of the 1.52 meter telescope (OHP). Two spectral regions, $4280-4720$ and 6150 - $7030 \AA$, were observed in 1996, while in 1997 we obtained data from 4095 to $8936 \AA$. In 1996 the dispersion in the red region was $33 \AA \mathrm{mm}^{-1}$ (resolution $\sim 1.1 \AA$ ) and $16.5 \AA \mathrm{mm}^{-1}$ (resolution $\sim 0.6 \AA$ ) in the blue region. In 1997 the dispersion was $33 \AA \mathrm{mm}^{-1}$ (resolution $\sim 1.2 \AA$ ) throughout the whole spectrum. A thorium-argon hollow cathode provided the wavelength calibration spectra. The flux calibration was completed by comparing observations of HR 7174 (HD 176318) to its absolute fluxes obtained by Gunn \& Stryker (1983). Two spectral regions (6275-7150 and $5590-6500 \AA$ A) were observed during cloudy weather which led to a wrong estimate of the fluxes. In these cases, the spectra were shifted until their fluxes coincide to those observed in the other regions. In Table 1 we report the integrated fluxes for each line appearing in the 1997 spectra.
The red part of the spectrum is strongly affected by telluric absorption bands. In view to identify possible strong transitions appearing in the polluted spectral regions, we divided the spectrum of MWC 314 by the spectrum observed for HR 7174 during the same nights. We emphasize that these "corrected" regions allow us only to identify possible strong transitions. The derived equivalent widths and intensities have to be used very carefully.

The July 1997 spectrum was taken with the CCD equipped echelle-spectrometer LYNX (Klochkova 1995) mounted at the Nasmyth focus of the 6 -meter telescope in the spectral range from 5370 to $6670 \AA$ A. A $1140 \times 1170$ pixels CCD was employed. We used an echelle-grating with the blaze angle of $64.3^{\circ}$ and $37.5 \mathrm{gr} \mathrm{mm}^{-1}$, the cross disperser has $300 \mathrm{gr} \mathrm{mm}^{-1}$ at first order. The July observations were obtained at the poor weather conditions (seeing approximately $3-4^{\prime \prime}$, clouds), therefore the slit size was enlarged to increase the signal-to-noise ratio. The average spectral resolution was equal to $0.4 \AA$. The November spectrum was obtained at the prime focus of the 6 -meter telescope with the echelle-spectrometer PFES (Panchuk et al. 1998) in the range from 4700 to $8590 \AA$. The seeing was nearly $2^{\prime \prime}$, while the spectral resolution was about $0.8 \AA$. However, due to the larger exposure time the signalto-noise ratio was larger than that in July. This allowed us to measure the line profiles more precizely, but led to the $\mathrm{H} \alpha$ saturation.

An argon-filled thorium hollow-cathode lamp was used as the source of comparison spectrum. Control and correction of instrumental displacement of the comparison and object spectra were done in using the telluric lines of $\mathrm{O}_{2}$ and $\mathrm{H}_{2} \mathrm{O}$. We tested focusing with the spectra obtained over the observing night using telluric lines. The sky spectrum did not reveal any splitting of these lines.

\section{Results}

\subsection{Line identifications}

The spectra have been normalized using spectral parts free of emission and absorption lines. Identification of the spectral lines was done on the basis of a recent catalog of Coluzzi (1993), a compilation of Johansson (1978) for Fe II lines, and a paper by Quinet et al. (1996) for [Fe II] lines. A comparison of our data obtained with different resolutions in the same spectral intervals shows that they are very similar. Because of this, the data obtained with higher resolution and/or higher signal-to-noise ratio was used for identification.

In totaly 406 emission lines were identified in the spectral range between 4197 and $8864 \AA$. Their observed wavelengths, identifications, peak intensities, equivalent widths, and integrated fluxes (for the lines detected in the OHP 1997 spectrum) are presented in Table 1.

The 63 absorption lines detected in the spectrum seem to be of photospheric origin, they have not been observed 

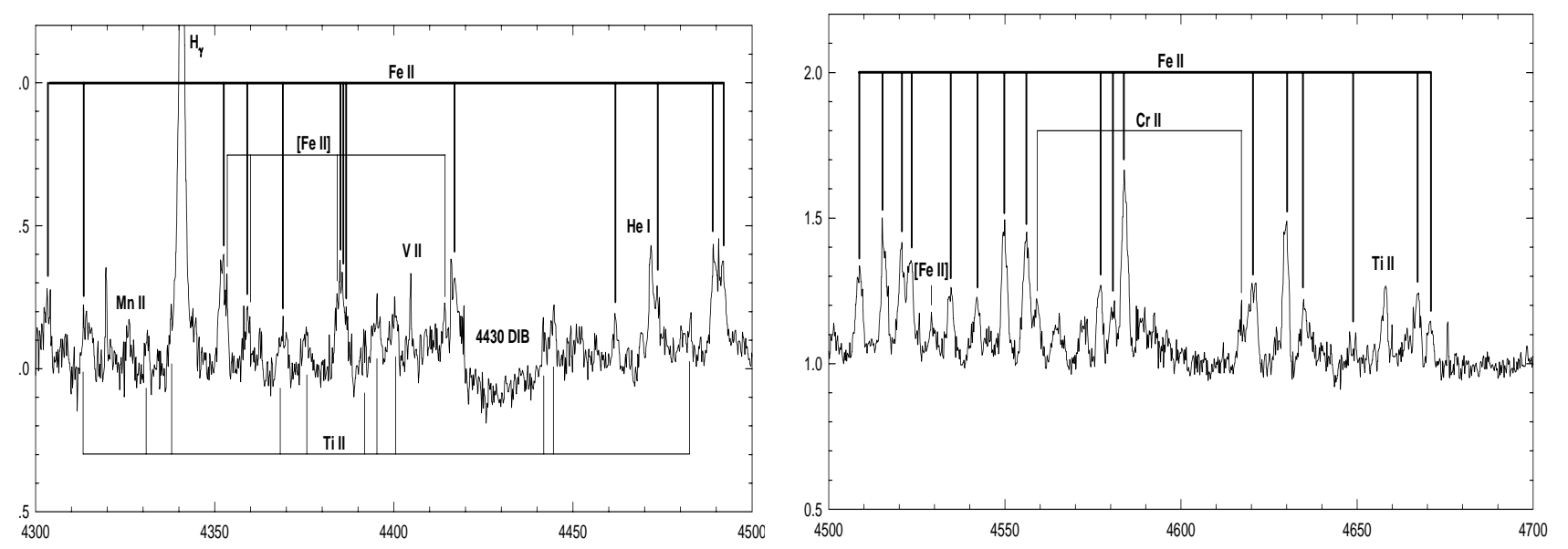

Fig. 1. The 1996 OHP spectrum of MWC 314 with identified lines. The wavelengths are given in Angströms, the intensity is normalized to the continuum level
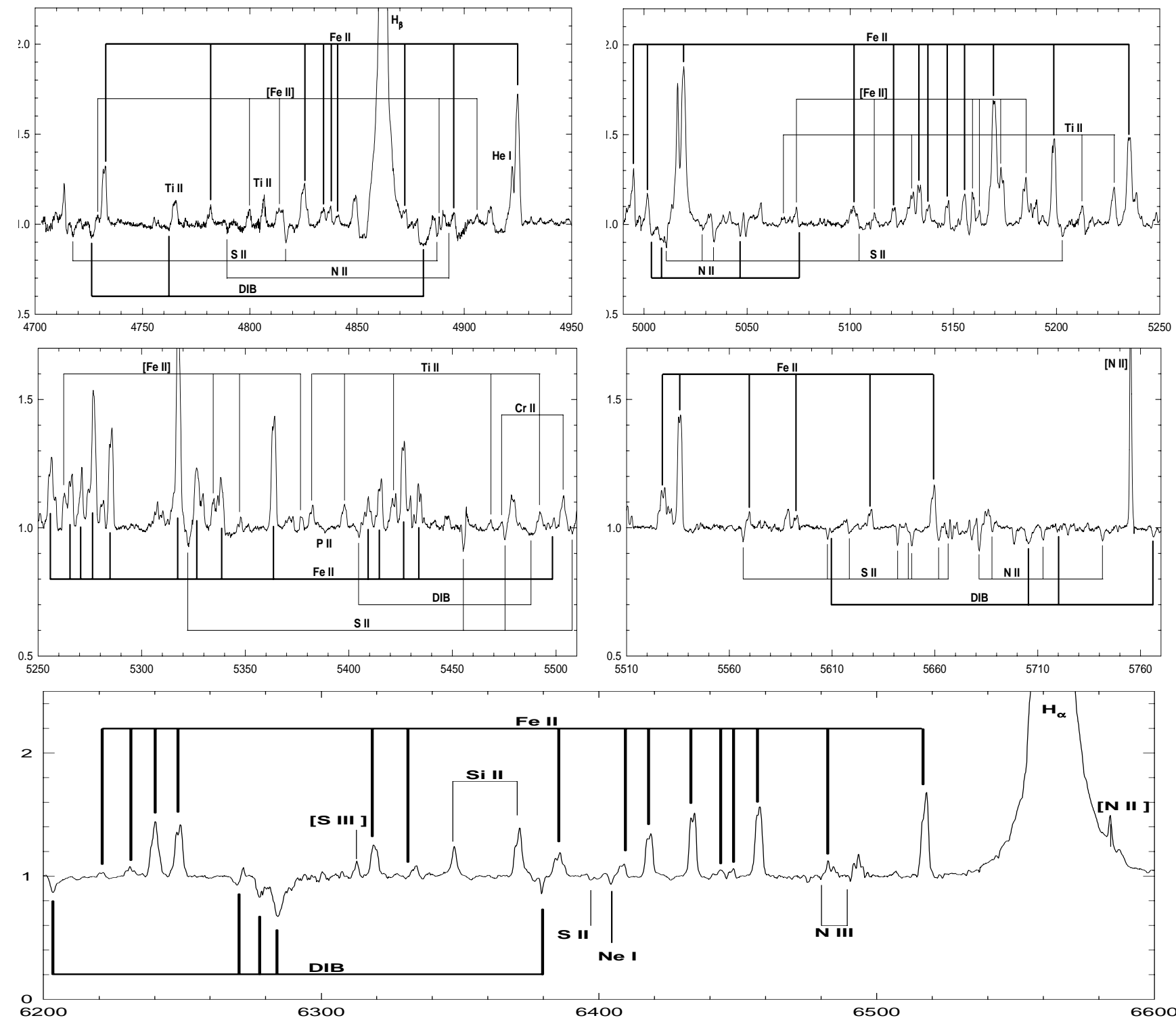

Fig. 2. The 1997 November SAO spectrum of MWC 314 with identified lines. Units of the wavelengths and intensities are the same as in Fig. 1 

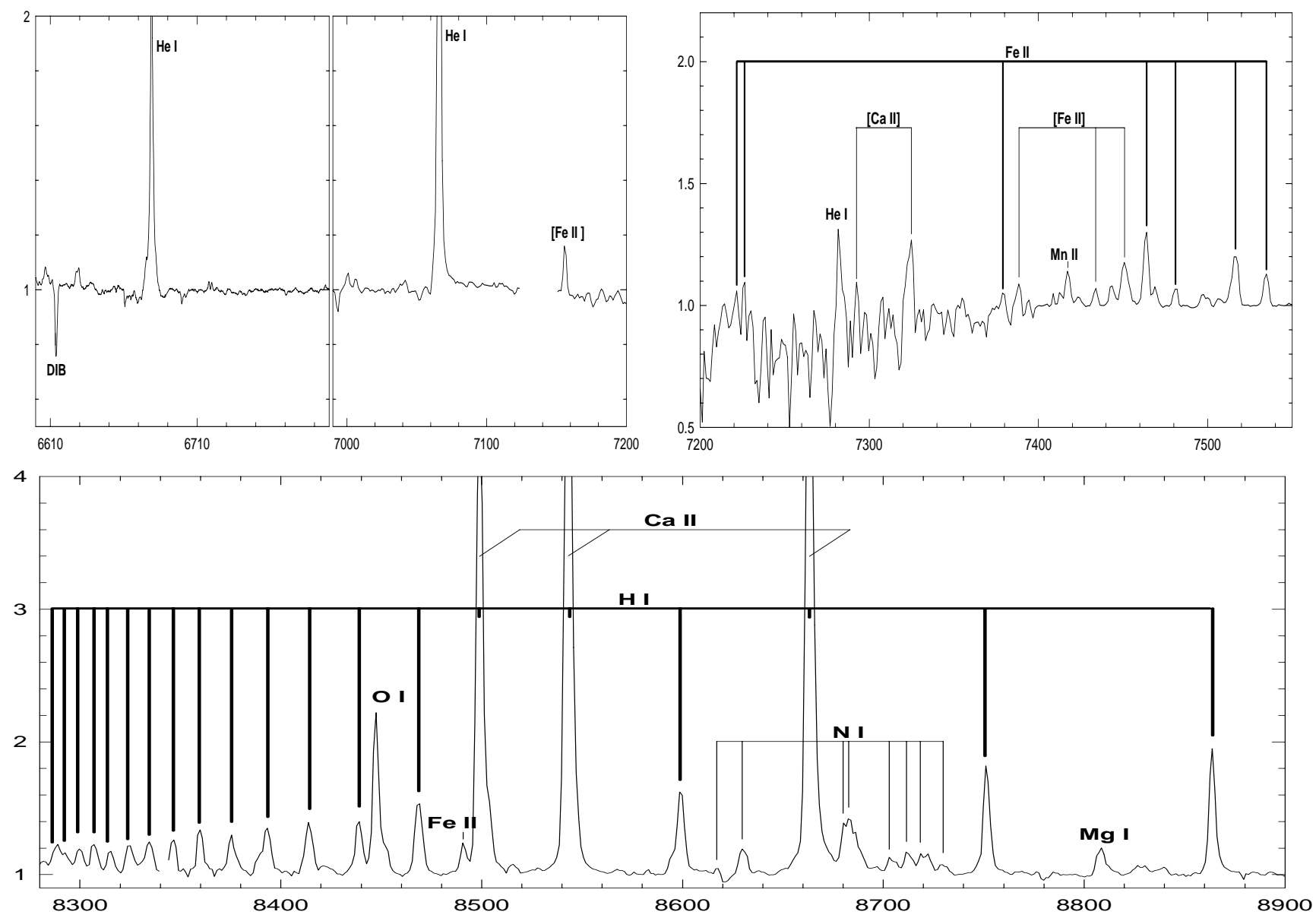

Fig. 3. The 1997 OHP spectrum of MWC 314 with identified lines. Units of the wavelengths and intensities are the same as in Fig. 1

previously. They are listed in Table 2. We found 60 absorption features (Table 3), which were identified with diffuse interstellar bands (Jenniskens \& Désert 1994). All of them are rather strong confirming a heavy reddening already indicated by the color-indices. We did not attempt estimating $E_{B-V}$ from their equivalent widths because of large scattering in corresponding relationships (e.g., Herbig 1975) and the fact that MWC 314 is one of the most reddened stars for which diffuse bands have been measured. At the same time, we have to note that the resolution of our spectra reveals many details of the diffuse features which can be used in their future statistical studies. Seven unidentified lines are listed in Table 4. Data on some photospheric lines of MWC 314 and several other supergiants are presented in Table 5 . Radial velocities of the spectral lines were measured by matching their direct and mirrored profiles. Mean radial velocities determined for the lines of different elements are presented in Table 6. Parts of the whole spectrum obtained at different time are presented in Figs. 1-3.

\subsection{Characteristics of the spectral lines}

Hydrogen. Three emission lines of the Balmer series were observed in our spectra, the Paschen lines are also seen in emission from $n=11$ up to $n=37$. The Balmer lines do not show any noticeable variability in comparison with the data obtained by Miroshnichenko (1996) in 1991. Even with the higher resolution spectra the $\mathrm{H} \alpha, \mathrm{H} \beta$, and $\mathrm{H} \gamma$ have no $\mathrm{P}$ Cyg-type absorptions. However, at a resolution better than $1 \AA \mathrm{H} \alpha$ and $\mathrm{H} \gamma$ appear double-peaked with a separation of 28 and $42 \mathrm{~km} \mathrm{~s}^{-1}$ respectively, which is near the resolution limit.

Helium. All the lines of neutral helium in our spectra are in emission and have a mean velocity of $21 \pm$ $3 \mathrm{~km} \mathrm{~s}^{-1}$, except for the strongest lines at 5876 and $7065 \AA$. The resolving power was not high enough to detect a possible double-peaked structure. It was suspected only for the $5876 \AA$ line. The lines at 4921, 5015, 5876, 6678, and $7065 \AA$ lines display shallow blue-shifted P Cyg-type absorptions $(\leq 0.15$ the continuum level) extended up to $\sim-300 \mathrm{~km} \mathrm{~s}^{-1}$ (Fig. 5). Very weak emission features are seen inside the absorption components. A very weak line of ionized helium at $4686 \AA$, which has not been reported 

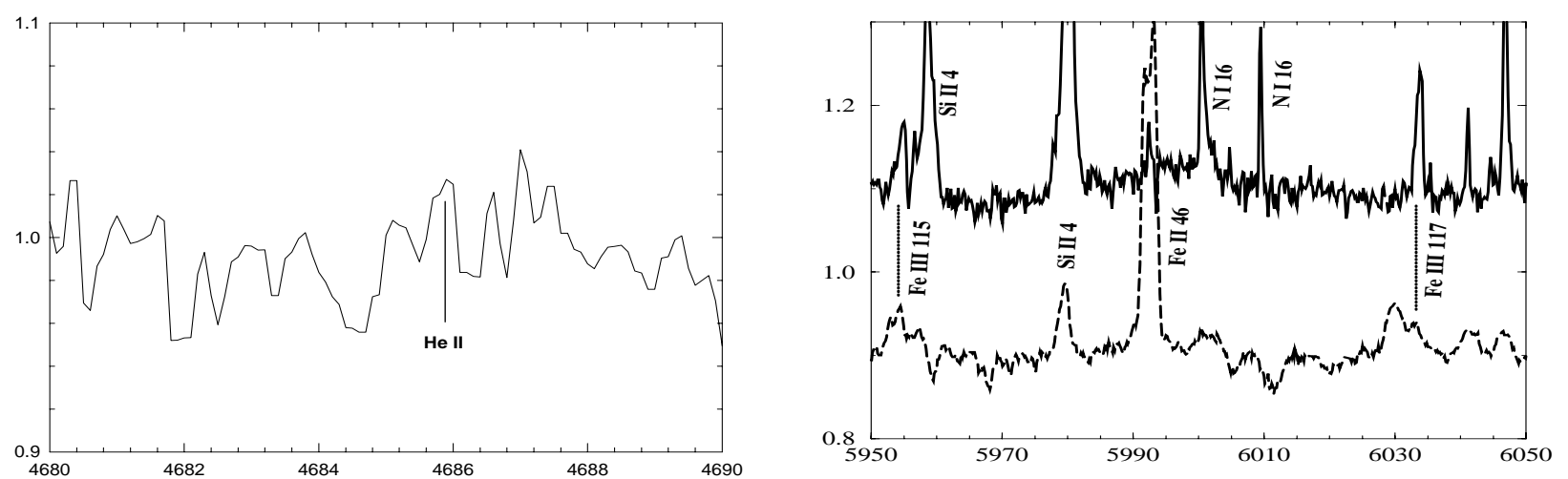

Fig. 4. Spectral parts containing the $\lambda 4686$ He II line (the 1996 OHP spectrum, left) and the Fe III lines (Mults. 115 and 117 , right). Solid line in the right part represents the spectrum of MWC 300 shown for comparison, dashed line represents a part of the November 1997 SAO spectrum of MWC 314. Units of the wavelengths and intensities are the same as in Fig. 1

before for MWC 314, is suspected to be present in the spectrum (Fig. 4).

Iron. This is the element, which exhibits the greatest number of lines. Almost all permitted lines of Fe II show a double-peaked structure. The separation of the peaks and their intensity ratio depend on the line strength. For the weak lines $\left(I_{\text {peak }} \leq 1.2 I_{\text {cont }}\right)$ they are $70 \mathrm{~km} \mathrm{~s}^{-1}$ and $0.65\left(I_{\text {blue }} / I_{\text {red }}\right)$, while for the strong lines $\left(I_{\text {peak }} \sim 1.5\right.$ $\left.I_{\text {cont }}\right) 40 \mathrm{~km} \mathrm{~s}^{-1}$ and 0.95 respectively (see also Sect. 4.1 ). 37 rather weak emission lines of [Fe II] were detected in comparison with only one line found by Miroshnichenko (1996). Eight permitted lines of doubly ionized iron and six [Fe III] lines were also identified.

Sodium. Only neutral sodium is present in the spectrum. Interstellar components of the $D_{1,2}$ lines of multiplet 1 are almost saturated indicating a very large reddening. Each of them consists of two components of almost equal intensity at $\sim+7$ and $+25 \mathrm{~km} \mathrm{~s}^{-1}$. At the same time, both lines contain emission components of circumstellar nature. The only line of multiplet 4 was found in pure emission in the red part of the spectrum.

Titanium and Chromium. These elements are mainly found in the form of singly ionized permitted lines and are, in general, weaker than the Fe II lines. Many of them are blended with the Fe II lines, which prevents determination of their profile shapes. Only in several cases are they undoubtfully double-peaked. Several lines of [Ti II] are seen in the red region, while no [Cr II] lines were identified.

Magnesium. Two emission lines of Mg II were detected near the red edge of the spectrum. Neutral $\mathrm{Mg}$ is present in emission as two rather strong lines of Mult.2 at 5173 and $5184 \AA$, the unresolved triplet at $8806.7 \AA$, and the doublet at $6319 \AA$, which is blended with a line of Fe II at $6318 \AA$. The latter was also found in the spectra of $\mathrm{B}[\mathrm{e}]$-supergiants in the Magellanic Clouds (Zickgraf et al. 1986).

Calcium. Calcium is represented by the singly ionized infrared triplet in emission which is much stronger than

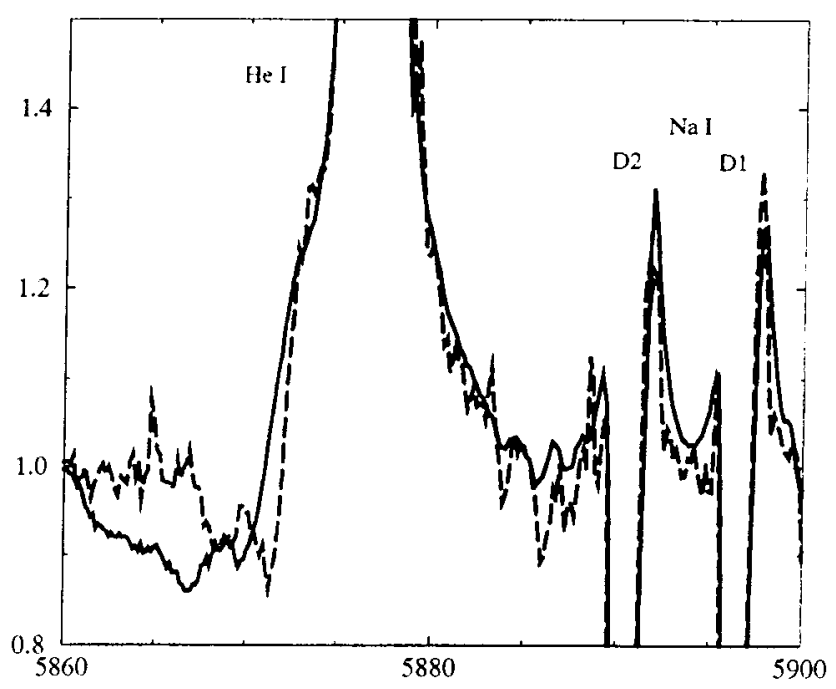

Fig. 5. A spectral part containing He I $5876 \AA$ line and sodium $D_{1,2}$ lines. The solid line represents the SAO spectrum obtained in July 1997, while the dashed line that of November 1997. Units of the wavelengths and intensities are the same as in Fig. 1

the Paschen lines blended with it. Two relatively weak emission lines of [Ca II] are also present in the red region.

Oxygen. Only five emission lines of neutral oxygen were detected in the spectrum. The permitted doublet at $8447 \AA$ is relatively strong $\left(I_{\text {peak }}=2.2 I_{\text {cont }}\right)$ while the permitted triplet at $7775 \AA$ is much weaker $\left(1.2 I_{\text {cont }}\right)$. The forbidden lines at 5577 and $6300 \AA$ are extremely weak.

Silicon. Six lines of Si II (multiplets 2, 4, and 5) were found in emission. The line at $6370 \AA$ is blended with a stronger Fe II double-peaked line, while the line at $6347 \AA$ is probably double-peaked, what can be revealed at higher resolution (Fig. 6).

Nitrogen. Several weak permitted emission lines of neutral nitrogen were found longward of $8600 \AA$. Forbidden 
emission lines are located at $5755 \AA$ (very strong line) and at both sides of $\mathrm{H} \alpha$. A number of $\mathrm{N}$ II absorption lines as well as three of $\mathrm{N}$ III were detected between 4197 and $6523 \AA$, which seemed to be photospheric.

Photospheric lines. Besides those of nitrogen, the photospheric lines of S II (26 lines), Ne I (8), C II (2), Al III (2), P II (1), and Si III (1) were detected. They have the same velocities as the stellar N II absorptions.

Other elements. Emission lines of singly ionized Mn (8 lines), V (4), Sc (3), Ba (1) were also found in the spectrum.

In our spectra we found almost all the emission lines reported by Swensson (1942). However, some of them might have different identifications than those suggested by Swensson. For example, a double-peaked emission centered at $4657.9 \AA$ is more likely due to a Ti II transition than to that of [Fe III] $4658.1 \AA$, because forbidden lines have single-peaked profiles in our spectra and positive radial velocities. Similarly, an emission at $5184 \AA$ is doublepeaked and is more likely to be a $\mathrm{Ti}$ II line of multiplet 86 rather than that of $\mathrm{Mg}$ I $5183.6 \AA$. The presence of the $\mathrm{Mg}$ II line in a blend at $4481-4482 \AA$, which probably consists of a Ti II (30) and a Fe II (20) line appearing in other blends as well, is uncertain.

Nearly 20 weak emission features (mainly with $\left.I / I_{\text {cont }}<1.05\right)$ can be identified with Fe I lines of multiplets 15, 60, 169. Some of them have double-peaked profiles and radial velocities similar to those of the permitted singly ionized metallic lines. Although this is not unexpected, we consider these identifications as doubtful because some strong lines of the mentioned multiplets are not seen in the spectrum (for example, Fe I (169) $\lambda 6252.6 \AA$ or Fe I $(60) \lambda 8688.6 \AA)$.

\subsection{Variations of the line intensities and profiles}

Variations of the spectral line intensities on a timescale of weeks were reported by Swensson (1942), who was going to analyse them later. However, this discussion was not published. Our observations show no significant variations of the line intensities or profiles, except for those in some very weak lines, which can be due to noise.

The profiles of the majority of the emission lines are very narrow $\left(\Delta v \sim 130 \mathrm{~km} \mathrm{~s}^{-1} \mathrm{FWHM}\right)$, which make their appearance dependent on the spectral resolution. However, comparison of our spectra obtained at different times but with the same resolution shows no significant changes (Fig. 6). The broad lines, like $\mathrm{H} \alpha$ (Fig. 7), reveal their fine structure at higher resolution but appear stable even on more extended period of time. Widths of some emission lines reported by Swensson (1942) are almost the same as those in our spectra. This fact along with the absence of significant photometric variability (Miroshnichenko 1996) allow to suggest a relative stability of the object's wind on a timescale of decades.

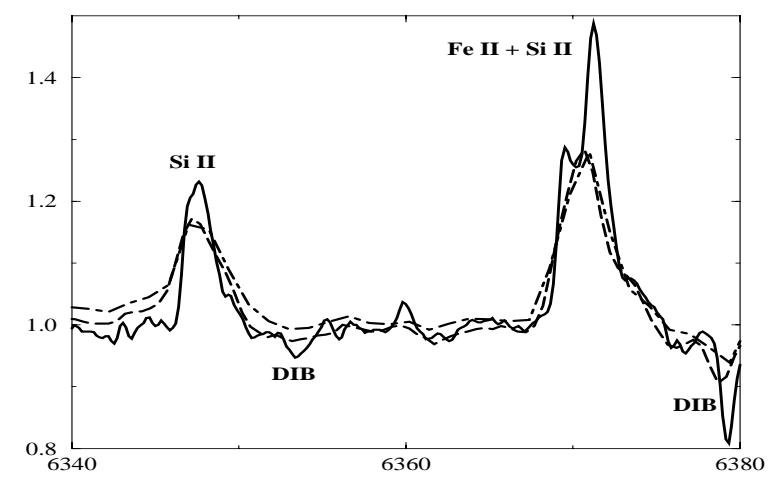

Fig. 6. Comparison of the observational data obtained at different time and with different spectral resolution. Solid line represents the July SAO spectrum, the OHP 1996 spectrum is shown by dashed line and the OHP 1997 spectrum by dotdashed line. Units of the wavelengths and intensities are the same as in Fig. 1

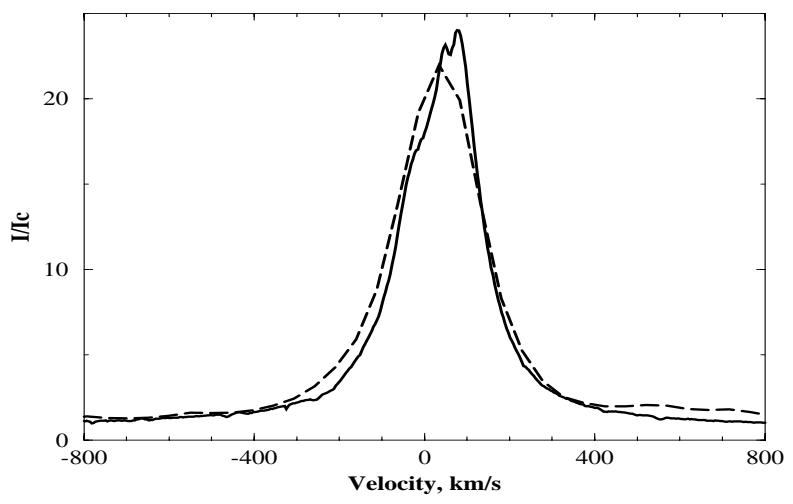

Fig. 7. Section of the spectrum around $\mathrm{H} \alpha$. The SAO 1997 spectrum is shown by solid line, the spectrum obtained by Miroshnichenko (1996) in 1991 at SAO by dashed line. Intensity is normalized to the continuum level

\section{Discussion}

There are not many objects in the Milky Way displaying so strong emission lines of H I and Fe II as MWC 314 does. Nearly all of them discovered so far have been recently collected in a catalog of Thé et al. (1994). Their physical parameters and evolutionary state may vary in a very large range from extremely massive relatively young supergiants (MWC 349) to low-mass proto-planetary nebulae (OY Gem). The nature of some of these stars, like MWC 84, MWC 930 (Miroshnichenko 1995), is still uncertain. However, the main characteristics of the stars and their environments are uncertain even for well-observed objects. One of the main problems causing incomplete knowledge of these objects is the lack of high-resolution spectroscopy and long-term photometric observations. Highresolution spectroscopy is important because the strong 
emissions of the circumstellar envelopes existing around such stars severely veil photospheric spectra. On the other hand, these observations help to constrain the shape of the gaseous component of the envelopes and to estimate distances toward the objects using radial velocities (e.g., Humphreys et al. 1989).

\subsection{Stellar wind}

In general, the emission line profiles observed in the spectra of MWC 314 are double-peaked without blue-shifted P Cyg-type absorptions. Such a structure is sharply different that of some other peculiar supergiants and LBVs like P Cyg, HD 316285, AG Car, while similar profiles are observed in such B[e] stars as MWC 349, MWC 84, OY Gem. This implies that the shape of the circumstellar envelope, where the emissions originate, deviates from spherical and that it is not viewed edge--on. Miroshnichenko (1996) modeled the MWC 314 Balmer line profiles in spherical geometry and converged theoretical profiles to match the observational resolution, which was about $2 \AA$ for his data. However, the results of his original calculations, which were done with a resolution of nearly $0.5 \AA$, always showed the presence of $\mathrm{P}$ Cyg-type absorption components. Now with a 5 times better resolution in the $\mathrm{H} \alpha$ region we still do not see any absorption component in the line profile. They are weak in the helium lines originating close to the stellar surface where density is significantly larger than in the region of the Balmer line formation. The same lines in the spectrum of $\mathrm{P}$ Cyg, which do not show evidence of a non-spherical wind (Najarro et al. 1997), have very strong absorption components. This strongly favors a nonspherical wind from the star. In a forthcoming paper we will model profiles of the hydrogen and helium lines using a disk-like matter distribution around MWC 314.

The weakness of the forbidden iron lines indicate that the stellar wind of MWC 314 does not contain extended low density regions. The widths of most emission lines are $130 \pm 5 \mathrm{~km} \mathrm{~s}^{-1} \mathrm{FWHM}$ and $160 \pm 5 \mathrm{~km} \mathrm{~s}^{-1}$ at the continuum level (FWZI). Only $\mathrm{H} \alpha$ and $\mathrm{H} \beta$ have FWZI of $\sim 1700$ and $900 \mathrm{~km} \mathrm{~s}^{-1}$ respectively which can be due to electron scattering effects. This is not surprising in such a dense wind with the physical parameters as quoted in the introduction. The strength of the Ca I IR triplet, which shows a larger intensity relatively to the continuum (4.35.8) among similar stars (Lopes et al. 1992; Andrillat et al. 1996, 1997; Jaschek et al. 1996a,b), is probably another evidence of the wind high density.

\subsection{Effective temperature}

Our new results confirmed the suggestion of Miroshnichenko (1996) that MWC 314 is an early B-type high-luminosity star. We detected its photospheric lines for the first time, however such well-known luminosity indicators as Si IV and O II lines have not been found in the spectrum. This is mainly due to the presence of a large number of emissions located at the positions of many photospheric lines, abundance anomalies (see below), and a limited spectral range of the obtained spectra. On the other hand, Fe III emission lines of multiplets 115 and 117 are usually observed in the spectra (Fig. 4) of early B-type hypergiants with $M_{V} \leq 8.0$ (Wolf \& Stahl 1985). These lines have radial velocities similar to those of Fe II and [Fe II], which are formed in the stellar wind. Nevertheless, the Fe III lines are narrower than the other iron lines and always single-peaked, indicating a different formation zone, which might be the polar regions of the non-spherical stellar wind (see a model for $\mathrm{B}[\mathrm{e}]$ supergiants of the Magellanic Clouds by Zickgraf et al. 1986).

The weak signes of He II lines in the spectrum (their presence needs further confirmation at a higher resolution and signal-to-noise ratio) constrains an upper level of $T_{\text {eff }}$ at nearly $27000 \mathrm{~K}$ (Schmutz et al. 1991). The presence of other high excitation lines, like [Fe III] and [S III], also point out to an early-B spectral type.

The photospheric lines can be seen at a resolution of less than $1 \AA$. Our list of photospheric absorptions identified in the spectrum of MWC 314 does not contain oxygen lines. The same situation has been previously reported for such LBVs as AG Car and HR Car (Hutsémekers \& van Drom 1991) and is suggested to be due to a N/O overabundance in the atmosphere. Other similar stars (e.g., $\mathrm{P}$ Cyg and MWC 300) show O II photospheric lines along with those of N II. In any case, such anomalies imply that MWC 314 is a highly evolved star. The presence of the $\mathrm{Ne}$ I lines gives this suggestion further support.

In order to estimate $T_{\text {eff }}$ of MWC 314 we compared characteristics of some of its photospheric lines showing dependence on stellar temperature with those of other supergiants. An atlas by Lennon et al. (1992) was used in the blue region $(\lambda \lambda 4200-4900 \AA)$, while spectra with echelle-spectrometers of the 1-meter SAO telescope (Musaev 1993, 1996) were examined in the red region $(\lambda \lambda 5400-6500 \AA)$. Both data sets were obtained with a resolution close to those of our data, i.e. $\sim 0.3-0.8 \AA$. Nearly 20 stars of spectral types O9 - B8 was used from the atlas and 7 B2 - B8 stars from the SAO archive.

In the blue region we found only one line, $\mathrm{N}$ II (M. 5) at $4630.5 \AA$, for the $T_{\text {eff }}$ estimate. Its strength increases with $T_{\text {eff }}$ decrease for the spectral types O9 - B2, then decreases towards B4, and remains weak and almost constant at lower temperatures. This gives a spectral type for MWC 314 of B0 or B3.5. Two lines of S II (M. 6) at 5453.8 and $5473.6 \AA$, two lines of N II (M. 3) at 5679.6 and 5686.2 $\AA$, four lines of Ne I (Ms. 1 and 5) at 6143.1, 6163.6, 6334.4, $6402.3 \AA$ and two lines of Al III (M. 2) at 5696.5 and $5722.7 \AA$ were used in the red region. The $\mathrm{N}$ II and $\mathrm{Al}$ III lines weaken sharply from B2 to B8, while the other lines used strengthen gradually from B2 to B5 remaining stable 
Table 5. Photospheric line characteristics in MWC 314 and other supergiants

\begin{tabular}{|c|c|c|c|c|c|c|c|c|c|c|c|c|c|c|c|c|}
\hline \multirow[t]{2}{*}{ Line } & \multicolumn{2}{|c|}{ MWC 314} & \multicolumn{2}{|c|}{$\begin{array}{l}\text { HD } 41117 \\
\text { B1.8 (1) }\end{array}$} & \multicolumn{2}{|c|}{$\begin{array}{c}\text { HD } 224055 \\
\text { B2.9 (2) }\end{array}$} & \multicolumn{2}{|c|}{$\begin{array}{l}\text { HD } 164353 \\
\text { B3.6 (1) }\end{array}$} & \multicolumn{2}{|c|}{$\begin{array}{l}\text { HD } 58350 \\
\text { B3.9 (3) }\end{array}$} & \multicolumn{2}{|c|}{$\begin{array}{l}\text { HD } 168625 \\
\text { B5.5 (3) }\end{array}$} & \multicolumn{2}{|c|}{$\begin{array}{c}\text { HD } 183143 \\
\text { B7.7 (3) }\end{array}$} & \multicolumn{2}{|c|}{$\begin{array}{c}\text { HD } 34085 \\
\text { B8.1 (3) }\end{array}$} \\
\hline & Int. & EW & Int. & EW & Int. & EW & Int. & EW & Int. & EW & Int. & EW & Int. & EW & Int. & EW \\
\hline SII 5453 & 0.88 & 0.15 & 0.97 & 0.06: & 0.87 & 0.16 & 0.84 & 0.15 & - & 0.15 & 0.83: & $0.2:$ & 0.80: & $0.3:$ & 0.85 & 0.16 \\
\hline SiI 5473 & 0.91 & 0.07: & 0.98 & 0.03: & 0.95 & 0.06 & 0.94 & 0.05 & - & 0.04 & 0.87: & - & 0.96 & 0.05: & 0.95 & 0.04 \\
\hline SII 5640 & 0.88 & 0.11 & 0.97 & 0.04 & 0.88 & 0.16 & 0.84 & 0.19 & - & 0.25 & 0.80: & - & - & - & 0.85 & 0.17 \\
\hline Niı 5679 & 0.89 & 0.13 & 0.66 & 0.63 & 0.80 & 0.28 & 0.89 & 0.12 & - & 0.14 & 0.88: & $0.2:$ & 0.93 & 0.08 & 0.96 & 0.05 \\
\hline NiI 5686 & 0.91 & 0.07 & - & - & 0.90 & 0.12 & 0.97 & 0.03 & - & 0.06 & $0.95:$ & - & 0.98 & 0.02: & - & - \\
\hline Al III 5696 & 0.94 & 0.09 & 0.83 & 0.28 & 0.85 & 0.21 & 0.89 & 0.11 & - & 0.12 & 0.88: & 0.08: & 0.95 & 0.07 & 0.95 & 0.05 \\
\hline Al III 5722 & 0.96 & 0.06: & 0.89 & 0.17 & 0.89 & 0.13 & 0.93 & 0.07 & - & 0.07 & 0.90: & 0.08: & 0.97 & 0.04 & 0.97 & 0.03 \\
\hline NeI 6143 & 0.91 & 0.10 & 0.99 & - & 0.95 & 0.06 & 0.94 & 0.07 & - & - & 0.95: & - & 0.95 & 0.07: & 0.95 & 0.07 \\
\hline NeI 6163 & 0.93 & 0.08 & - & - & - & - & 0.98 & 0.02 & - & - & 0.97: & - & - & - & 0.97 & 0.03 \\
\hline NeI 6334 & 0.92 & $0.11:$ & - & - & 0.97 & 0.04 & 0.96 & 0.05 & - & 0.08 & 0.95 & $0.05:$ & 0.97: & 0.04: & 0.94 & 0.05 \\
\hline NeI 6402 & 0.86 & 0.20: & 0.97 & 0.08 & 0.91 & 0.15 & 0.88 & 0.13 & - & 0.16 & 0.90 & 0.14: & $0.88:$ & $0.16:$ & 0.90 & 0.13 \\
\hline
\end{tabular}

The peak intensities are given in units of the continuum while the equivalent widths in Angströms. For each of the comparison stars their spectral types and references to them are given. The references are as follows: 1 - Kopylov (1958); 2 - Bartaya et al. (1994); 3 - Chentsov \& Luud (1989). Equivalent widths for HD 58350 were taken from Underhill \& Fahey (1973).

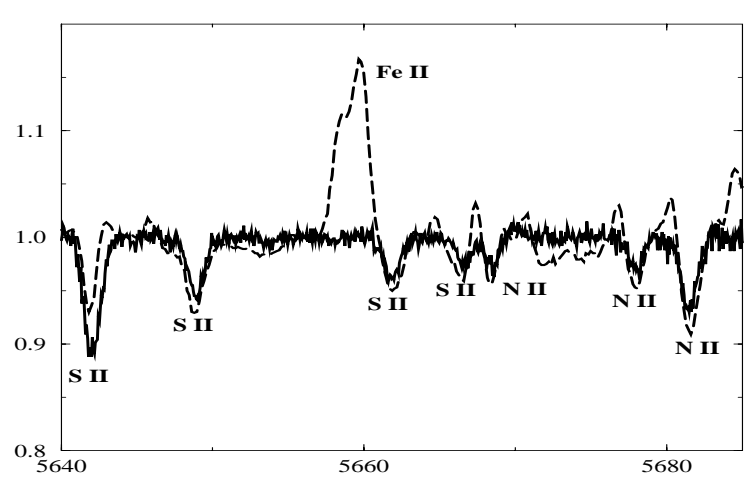

Fig. 8. Comparison of the photospheric lines in the spectra of MWC 314 (dashed line) and HD 183143 (B7 Ia, solid line). The spectrum of HD 183143 was taken at the 1-meter SAO telescope and shifted to the photospheric radial velocity of MWC 314. Units of the wavelengths and intensities are the same as in Fig. 1

with further temperature decrease. Spectra of two hotter stars, HD 190603 (B1.5) and HD 38771 (B0.4), obtained at $\mathrm{SAO}$ were also examined. They contain the very weak $\mathrm{S}$ II lines and only traces of the Ne I lines and were not included in Table 5 . The Ne I lines give a spectral type of B2.5 for NWC 314, N II and S II - B3.5, while Al III - B4-5. An example of such a comparison is presented in Fig. 8.

Thus, the photospheric lines characteristics suggest that MWC 314 has a spectral type of nearly B3 or B0, fixing the lower limit of $T_{\text {eff }}$ at approximately $15000 \mathrm{~K}$. This value seems to be rather low and inconsistent with appearance of the strong He I emission lines, which are not observed in B3 stars (e.g., Zickgraf et al. 1986). Furthermore, Walborn (1980) noted that a Si III (M. 4) line at $5739.8 \AA$, which is seen in the MWC 314 spectrum, appears only in early-B stars (B0.5 and B1.5). Additionally, Miroshnichenko (1996) showed that $T_{\text {eff }}<$ $20000 \mathrm{~K}$ is less consistent with the SED of MWC 314 in the UV region than 25000 and $30000 \mathrm{~K}$.

On the other hand, B0-B1 supergiants display a strong blend of $\mathrm{C}$ III lines at $4647-4650 \AA$, which is not evident in MWC 314 because of veiling by a blend of the Mn II and Fe II emissions. Furthermore, the absence of the $\mathrm{C}$ III lines can also be explained by a carbon depletion. Thus, the underabundance of oxygen and carbon combined to the overabundance of nitrogen could be an evidence of $\mathrm{CNO}$ processing. In this case, a helium enrichment of the photosphere is expected. The Ne I lines in MWC 314 are stronger than in any other supergiant taken for comparison (Table 5). The B0-B1 supergiants do not show these lines. This feature of MWC 314 might be due to its larger mass $\left(\sim 80 M_{\odot}\right.$, Miroshnichenko 1996) and, hence, faster evolution. Thus, B0, corresponding to $T_{\text {eff }} \sim$ $25000 \mathrm{~K}$, fits the whole picture of the MWC 314 spectrum better.

\subsection{Radial velocities, distance, luminosity}

The spectral resolution of our data allows to study radial velocities of different lines. Only the lines with clearly resolved peaks uncontaminated by nearby features having comparable intensities have been selected for radial velocity measurements. It is seen from Table 6 that there is a significant difference in velocities of different types of lines. The heliocentric velocities of the photospheric lines are grouped around $+81 \mathrm{~km} \mathrm{~s}^{-1}$, those of the lines of ionized $\mathrm{Fe}, \mathrm{Ti}$, and $\mathrm{Ca}$ as well as of the Balmer lines around $+41 \mathrm{~km} \mathrm{~s}^{-1}$, while the lines of He I and Si II have 
Table 6. Radial velocities

\begin{tabular}{|c|c|c|}
\hline Element & Velocity & $N$ \\
\hline Balmer lines $(\mathrm{H} \alpha, \mathrm{H} \beta, \mathrm{H} \gamma)$ & $+45 \pm 3$ & 3 \\
\hline paschen lines (P16-P22) & $+34 \pm 3$ & 7 \\
\hline $\mathrm{He} \mathrm{I}^{\mathrm{a}}$ & $+21 \pm 3$ & 7 \\
\hline $\mathrm{He} \mathrm{I}^{\mathrm{b}}$ & $+32:$ & 2 \\
\hline$[\mathrm{Ca}$ II $]$ & $+28:$ & 2 \\
\hline Permitted metallic lines ${ }^{\mathrm{c}}$ & $+40.5 \pm 0.3$ & 33 \\
\hline Forbidden metallic lines ${ }^{\mathrm{d}}$ & $+15 \pm 2$ & 20 \\
\hline Si II & $+17 \pm 1$ & 4 \\
\hline Na I emissions & $+37:$ & 2 \\
\hline $\mathrm{Na}$ I absorptions & $+13:$ & 2 \\
\hline $\mathrm{S}, \mathrm{N}$ II, Ne I & $+81 \pm 1$ & 60 \\
\hline DIBs & +4 & 60 \\
\hline
\end{tabular}

a all detected lines besides those at $\lambda 5876$ and $7065 \AA$,

b only the lines at $\lambda 5876$ and $7065 \AA$,

c Fe II, Ti II, Cr II,

d all forbidden lines except [Ca II],

Radial velocities are given in $\mathrm{km} \mathrm{s}^{-1}$, numbers of the lines used to determine the mean velocity are given in the last column.

velocities close to $+20 \mathrm{~km} \mathrm{~s}^{-1}$. The paschen lines, those of Ca II, the strongest Fe II (mainly of Mult. 42) lines, He I 5876 and $7065 \AA$ lines, and emission components of $\mathrm{Na}$ I D-lines have radial velocities close to $+35 \mathrm{~km} \mathrm{~s}^{-1}$. The velocities of the diffuse interstellar bands are close to zero. The correction for the solar motion in the Galaxy, which should be applied to these values to reduce them to the local standard of rest, is $+14 \mathrm{~km} \mathrm{~s}^{-1}$.

These data can be used to estimate the systemic velocity and, finally, distance toward the object employing differential rotation of the Galaxy. To do this the systemic velocity should be separated from other motions. The photospheric lines represent radial velocity of the star itself which might have its own peculiar motion. The lines of $\mathrm{He}$ and $\mathrm{Si}$ are formed in the very inner parts of the wind and their velocities can be affected by strong acceleration of the outflowing matter close to the star. The lines of other metals originate at larger distances from the star and most probably have radial velocities close to that of the system. The hydrogen lines forming in a very extended region of the wind have mean velocities comparable to those of the ionized metals. Taking into account the correction to the local standard of rest we can estimate the systemic velocity as $+55 \pm 2 \mathrm{~km} \mathrm{~s}^{-1}$. To calculate relation between distance $(D)$ from the Sun and radial velocity in the object's direction we used a second order expansion of the standard equation for circular rotation with $R_{0}=8.5 \mathrm{kpc}$, $A_{1}=16.1 \mathrm{~km} \mathrm{~s}^{-1} \mathrm{kpc}^{-1}$, and $A_{2}=-0.7 \mathrm{~km} \mathrm{~s}^{-1} \mathrm{kpc}^{-1}$ (Dubath et al. 1988). The systemic velocity corresponds to $D=3.0 \pm 0.2 \mathrm{kpc}$, which is in excellent agreement with the previous estimate of Miroshnichenko (1996).
We should note that this method can be applied only to the objects having wind velocities much larger than those measured for the star itself using photospheric lines. If an object has additional components of its motion (e.g., orbital motion in a binary system) the problem becomes much more complicated. This method has already given reasonable results for such $\mathrm{LBV}$ candidates as AG Car (Humphreys et al. 1989), HR Car (Hutsémekers \& van Drom 1991), and He3-519 (Smith et al. 1994). However, in other cases one should be careful estimating distance using radial velocities and the galactic rotation curve. For example, for MWC $300\left(v_{\text {rad }}=26 \pm 3 \mathrm{~km} \mathrm{~s}^{-1}\right.$, Winkler \& Wolf 1989) it gives $D=2.1 \mathrm{kpc}$, which is inconsistent with hypergiant classification for the star. In our case the estimate is confirmed by a strong reddening, complex structure of the $\mathrm{Na}$ I $D_{1,2}$ lines, and the relation between $A_{V}$ and $D$ in the MWC 314 direction (Miroshnichenko 1996).

With our refined values of the distance and effective temperature we can now re-estimate stellar parameters of MWC 314. Since the spectrum contains many emission lines we have to correct the visual flux for their contribution in order to derive absolute visual magnitude of the star. This correction is not expected to be large and we calculate it to realize how strong it could affect the result. Taking into account 172 emission lines detected in a spectral region corrsponding to the Johnson $V$-band (Kornilov et al. 1991) we found that their net effect is only 0 m $^{\mathrm{m}} 05$ added to the continuum level. Using the mean level of the object's visual brightness $V=9.9$ and the value of interstellar extinction $A_{V}=5.6$ (Miroshnichenko 1996) in combination with $D=3 \mathrm{kpc}$ derived here one can calculate $M_{V}=-8{ }^{\mathrm{m}} 1$. The bolometric correction corresponding to $T_{\text {eff }}=25000 \mathrm{~K},-2.5$ (Gubotchkin \& Miroshnichenko 1991), gives an absolute bolometric magnitude $M_{\mathrm{bol}}=-10^{\mathrm{m}} 6$ or $\log L_{\mathrm{bol}} / L_{\odot}=6.1$, and, consequently, stellar radius $R_{*}=60 R_{\odot}$.

The accuracy of the luminosity and radius estimates are set by the uncertainties of $A_{V}, T_{\text {eff }}$, and $D$, which are approximately $10 \%$ each. The latter give an uncertainty of 0.25 in $M_{V}$, about $30 \%$ in $L_{\text {bol }}$, and $50 \%$ in $R_{*}$. A lower limit for $R_{*}$ is set by the $A_{V} \sim D$ relation in the vicinity of MWC 314 (Miroshnichenko 1996), which shows a constant increase of $A_{V}$ up to $2 \mathrm{kpc}$ from the Sun. At this minimum distance the star would have a radius of $50 R_{\odot}$. The upper level of $R_{*}, 90 R_{\odot}$, gives $\log L_{\mathrm{bol}} / L_{\odot}=6.5$ and, therefore, $D=4.3 \mathrm{kpc}$. An independent estimate of $D$ can be obtained by means of radio continuum observations. The radio flux at the lower $D$ limit would be 5 times larger than that at the upper one, while the expected value at 3 $\mathrm{kpc}$ is about $4 \mathrm{mJy}$ according to the model calculations of Miroshnichenko (1996).

\section{Conclusions and future work}

The new high-resolution spectra of MWC 314 obtained in a range from 4200 to $8936 \AA$ allow us to constrain 
main physical parameters of the star giving additional evidence that it is an early B-type hypergiant close to the Eddington limit. An unusual chemical composition deduced from the identified photospheric lines suggesting a N/O overabundance, which is similar to those of LBV stars AG and HR Car, also supports its status as an LBV candidate. The lack of a surrounding nebula points out that the star has not experienced any eruption yet or it happened a long time ago. Coronographic observations would help to distinguish between the two possibilities (e.g., Nota et al. 1995).

The double-peaked shapes and the absence of the blue-shifted absorption components in most of the emission lines imply a non-spherical geometry of the stellar wind. The emission-line spectrum of MWC 314 is similar to those of B[e] supergiants, such as Hen S22, Hen S134, R126, having almost the same stellar parameters and nonspherical winds (Zickgraf et al. 1986). In contrast to these objects, at present MWC 314 does not show any infrared excess due to radiation of circumstellar dust, however, one can expect a dust formation event around MWC 314 in the future. In this case the observed lack of oxygen would imply the absence of the silicate features at 10 and 18 mum as in the infrared spectra of the $\mathrm{B}[\mathrm{e}]$ supergiants (Roche et al. 1993).

Thus, our present study confirms that MWC 314 is one of the most luminous stars in the Milky Way and shows importance of its high-resolution spectroscopy. Besides addition of a new information about the star and its wind it allows us to outline directions of the object's future investigation. High-resolution spectroscopic observations are needed to:

1. look for the photospheric features in the blue region (e.g., absorption lines of C III, Si III, Si IV) which can be used to refine the stellar parameters,

2. look for the spectral line variability, which is expected in such a luminous object,

3. check whether He II lines are present or not.

Finally, we would like to emphasize that other types of observations suggested by Miroshnichenko (1996) are also important. For example, photometric monitoring is capable of revealing outbursts which can be expected from this LBV candidate at any time. Radio observations in continuum can help to ascertain our distance estimate.

Acknowledgements. We are grateful to D.J. Lennon for making his high-resolution atlas of supergiants available to us in numerical form and to O. Stahl for supplying us with the spectrum of MWC 300. We wish also to thank the referee for his comments and suggestions which improved our paper.

\section{References}

Allen D.A., 1973, MNRAS 161, 145

Andrillat Y., Jaschek M., Jaschek C., 1996, A\&AS 118, 495

Andrillat Y., Jaschek C., Jaschek M., 1997, A\&AS 124, 441

Bartaya R.A., Chargeishirli K.B., Chentsov E.L., Shkagosheva Z.V., 1994, Bull. Spec. Astrophys. Obs. 38, 103

Chentsov E.L., Luud L.S., 1989, Astrofizika 31, 5

Coluzzi R., 1993, Bull. Inf. CDS 43, 7

Dubath P., Mayor M., Burki G., 1988, A\&A 205, 77

Gillet D., Burnage R., Kohler D., et al., 1994, A\&AS 108, 181

Gubotchkin A.N., Miroshnichenko A.S., 1991, Kinematics Phys. Celest. Bodies 7, 64

Gunn J.E., Stryker L.L., 1983, ApJS 52, 121

Herbig G.H., 1975, ApJ 196, 129

Hiltner W.A., 1956, ApJS 2, 389

Humphreys R.M., Lamers H.J.G.L.M., Hoekzema N., Cassatela A., 1989, A\&A 218, L17

Hutsémekers D., van Drom E., 1991, A\&A 248, 141

Jaschek C., Andrillat Y., Jaschek M., 1996a, A\&AS 117, 281

Jaschek M., Andrillat Y., Jaschek C., 1996b, A\&AS 120, 99

Jenniskens P., Désert F.-X., 1994, A\&AS 106, 39

Johansson S., 1978, Phys. Scr. 18, 217

Klochkova V.G., 1995, Echelle-spectrometer LYNX. User Manual. Spec. Astrophys. Obs. Technical Report No. 243

Kopylov I.M., 1958, Izv. Krym. Astrophys. Obs. 20, 156

Kornilov V.G., Volkov I.M., Zakharov A.I., et al., 1991, Sternberg Astron. Inst. Proc., v. 63

Kurucz R.L., 1979, ApJS 40, 1

Lennon D.J., Dufton P.L., Fitzsimmons A., 1992, A\&AS 94, 569

Merrill P.W., 1927, ApJ 65, 286

Miroshnichenko A.S., 1995, A\&ATr 5, 251

Miroshnichenko A.S., 1996, A\&A 312, 941

Musaev F.A., 1993, Pis'ma v Astron. Zh. 19, 776

Musaev F.A., 1996, Pis'ma v Astron. Zh. 22, 795

Najarro F., Hillier D.J., Stahl O., 1997, A\&A 326, 1117

Nota A., Livio M., Clampin M., Schulte-Ladbeck R.E., 1995, ApJ 448, 788

Panchuk V.E., et al., 1998, Bull. Spec. Astrophys. Obs., v. 44 (in press)

Quinet P., Le Dourneuf M., Zeippen C.J., 1996, A\&AS 120, 361

Roche P.F., Aitken D.K., Smith C.H., 1993, MNRAS 262, 301

Schmutz W., Leitherer C., Hubeny I., Vogel M., Hamann W.R., Wessolowski U., 1991, ApJ 372, 664

Smith L.J., Crowther P.A., Prinja R.K., 1994, A\&A 281, 833

Swensson J.W., 1942, ApJ 97, 226

Thé P.S., de Winter D., Pérez M.R., 1994, A\&AS 104, 315

Underhill A.B., Fahey R.P., 1973, ApJS 25, 463

Walborn N.R., 1980, ApJS 44, 353

Winkler H., Wolf B., 1989, A\&A 219, 151

Wolf B., Stahl O., 1985, A\&A 148, 412

Zickgraf F.J., Wolf B., Stahl O., Leitherer C., Appenzeller I., 1986, A\&A 163, 119 